\title{
Laser-induced deposition of nanostructured copper tracks from solutions containing oxidising additives (withdrawal notice)
}

Sergey Fateev, Svetlana Araslanova, Vasilii Mironov, Dmitrii Gordeychuk

Sergey A. Fateev, Svetlana M. Araslanova, Vasilii S. Mironov, Dmitrii I. Gordeychuk, "Laser-induced deposition of nanostructured copper tracks from solutions containing oxidising additives (withdrawal notice)," Proc. SPIE 9543, Third International Symposium on Laser Interaction with Matter, 95432A (4 May 2015); doi: 10.1117/12.2182077

Event: Third International Symposium on Laser Interaction with Matter, 2014, Jiangsu, China 


\title{
Laser-induced deposition of nanostructured copper tracks from solutions containing oxidising additives (withdrawal notice)
}

\author{
Sergey A. Fateev; Svetlana M. Araslanova ; Vasilii S. Mironov ; Dmitrii I. Gordeychuk
}

Saint Petersburg State Univ. (Russian Federation)

Proc. SPIE 9543, Third International Symposium on Laser Interaction with Matter, 95432A (May 4, 2015);

doi:10.1117/12.2182077

From Conference Volume 9543

Proc. SPIE 9543, Third International Symposium on Laser Interaction with Matter, 95432A (May 4, 2015); doi:10.1117/12.2182077

Online Publication Date: 4 May 2015

Withdrawn from Publication: 10 August 2015

This paper was withdrawn from the SPIE Digital Library on 10 August 2015 by the publisher upon verifying that significant portions of the paper were copied from the following publications without attribution or permission:

Dmitrii Semenok, "Application of lower aliphatic alcohols as reducing agents for increasing efficiency of the LCLD process," Proc. SPIE 9135, Laser Sources and Applications II, 91350X (May 1, 2014); doi: 10.1117/12.2049576;

and

Dmitriy Vladimirovich Semenok, Sergey Vladimirovich Safonov, Vladimir Kochemirovsky, "The Technology of Laser-Induced Deposition of Nanostructured Metallic Conductors on the Dielectric Substrate," in Proceedings of Nanomaterials: Application \& Properties '2012, Vol. 1 No 4,

04NMEEE05(2pp), the Crimea, Ukraine (September 2012);

http://nap.sumdu.edu.ua/index.php/nap/index/pages/view/ProcNAP

The citations are provided here so that interested readers can access the information from the original sources. 